\title{
ANÁLISIS DE LA EVOLUCIÓN ESPACIO-TEMPORAL DEL NDVI SOBRE ÁREAS VEGETADAS Y ZONAS DE RIESGO DE EROSIÓN EN EL PIRINEO CENTRAL
}

\author{
L. C. Alatorre ${ }^{1}$, S. Beguería ${ }^{2}$ \& S. M. Vicente-Serrano ${ }^{1}$ \\ 1 Instituto Pirenaico de Ecología (CSIC), Avda. Montañana 1005, 50059-Zaragoza. PO Box 13034, \\ 50080-Zaragoza (España) \\ 2 Estación Experimental de Aula Dei (CSIC), Avda. Montañana 1005, 50059-Zaragoza. PO Box 13034, \\ 50080-Zaragoza (España) \\ E-mail: lalatorre@ipe.csic.es \\ ABSTRACT.- The temporal evolution of vegetation activity on various land \\ cover classes in the Spanish Pyrenees was analyzed. Two time series of the normali- \\ zed difference vegetation index (NDVI) were used, corresponding to March (early \\ spring) and August (the end of summer). The series were generated from Landsat \\ TM and Landsat ETM+ images for the period 1984-2007. An increase in the NDVI \\ in March was found for vegetated areas, and the opposite trend was found in both \\ March and August for degraded areas (badlands and erosion risk areas). The rise in \\ minimum temperature during the study period appears to be the most important fac- \\ tor explaining the increased NDVI in the vegetated areas. In degraded areas, no cli- \\ matic or topographic variable was associated with the negative trend in the NDVI, \\ which may be related to erosion processes taking place in these regions.
}

Keywords: Landsat TM and ETM+; NDVI; Spanish Pyrenees; vegetation activity; badlands, erosion risk areas.

RESUMEN.- En este trabajo se ha analizado la evolución temporal y espacial de la dinámica vegetal sobre varias coberturas de suelo en el Pirineo central, España. Se han utilizado dos series temporales de NDVI, la primera corresponde al mes de Abril (inicio de primavera) y la segunda al mes de Agosto (final de verano). Las series fueron construidas a partir de imágenes Landsat TM y ETM+ para el periodo del 1984-2007. Los resultados muestran un incremento del NDVI en el mes de Marzo para las áreas vegetadas, mientras que las áreas degradadas (cárcavas y zonas de riesgo de erosión) presentaron una tendencia negativa del NDVI. El incremento de las temperaturas mínimas durante el periodo de estudio fue el factor más impor- 
tante para explicar el incremento del NDVI en las áreas vegetadas. En las áreas degradadas, no se encontró ninguna variable climática o topográfica que explicará la tendencia negativa del NDVI, lo cual se ha relacionado con los procesos de erosión acelerada que tienen lugar en la región.

Palabras clave: Landsat TM y ETM+; Pirineo central español; actividad vegetal; cárcavas; zonas de riesgo de erosión.

\section{Introduction}

Los estudios sobre la erosión se han realizado a diversas escalas espaciales, desde escalas locales a escalas regionales (VRIELING et al., 2006), proporcionado información de tipo cuantitativo (tasas de erosión) o cualitativo (p. ej. zonas de riesgo de erosión). De cara a la gestión medioambiental, resulta de gran interés la obtención de cartografías de zonas de riesgo de erosión, que permiten concentrar las actuaciones en los lugares donde éstas resultarán más adecuadas. Existe en la actualidad una gran diversidad de métodos para determinar la erosión, los cuales difieren grandemente en cuanto a la escala espacial y el propósito (WARREN, 2002).

Los métodos para evaluar el riesgo de erosión a escala de cuenca y regional $\left(10-10,000 \mathrm{~km}^{2}\right)$ incluyen la aplicación de modelos de erosión y aproximaciones cualitativas mediante el uso de teledetección (TLD) y sistemas de información geográfica (SIG). ALATORRE \& BEGUERÍA (2009a) hicieron una descripción muy exhaustiva sobre los modelos de erosión con los que se cuenta en la actualidad. Sin embargo, en la mayoría de los casos los modelos de erosión han sido creados para escalas pequeñas y su extrapolación a escalas más grandes (cuenca o regional) no resulta sencilla (KIRKBY et al., 1996). La TLD y los SIG han mostrado un gran potencial para la evaluación de la erosión a escalas regionales, incluyendo la identificación de áreas con erosión activa, la estimación de los factores que controlan la erosión, la monitorización del avance de la erosión a lo largo del tiempo, así como, el análisis de la dinámica vegetal y sus características (LAMBIN, 1996).

La dinámica vegetal tiene un papel muy importante en la evaluación de los procesos ambientales a causa de su estrecha relación entre la biósfera y los parámetros globales, incluyendo: la concentración de $\mathrm{CO}^{2}$ atmosférico (ZENG et al., 1999), la influencia de la vegetación en el ciclo hidrológico a escala local (BEGUERÍA et al., 2003), la estructura y diversidad del paisaje (OLSSON et al., 2000), los procesos de erosión y transporte de sedimentos (ALATORRE \& BEGUERÍA, 2009b). 
Varios estudios han identificado cambios en la dinámica vegetal a escala continental (DELBART et al., 2006), regional, y local (MARTÍNEZ-VILLALTA et al., 2008) en las últimas décadas. La mayoría de los cambios han sido causados por actividad humana, particularmente por la deforestación (DeFries et al., 2002) y los incendios forestales (RIAÑO et al., 2007), por otra parte, el abandono rural y la consiguiente marginalización de algunas regiones ha contribuido al proceso de recuperación vegetal (VICENTESERRANO et al., 2004). Sin embargo, numerosos estudios han encontrado un incremento en la actividad vegetal sobre distintos ecosistemas del mundo (LUCHT et al., 2002), sugiriendo que la causa principal son las variaciones en la precipitación y / o temperatura (DELBART et al., 2008).

En el Pirineo central español diferentes estudios han analizado las variaciones -temporales y espaciales de la actividad vegetal sobre distintas cuberturas vegetales a escala regional y local (VICENTE-SERRANO et al., 2004; VICENTE-SERRANO et al., 2006a, 2006b; LASANTA \& VICENTESERRANO, 2007), teniendo como objetivos: i) evaluar los cambios en la cubierta vegetal en los últimos 50 años; ii) detectar la tendencia global de la biomasa vegetal; iii) estudiar los cambios de la actividad foliar en regiones forestales; iv) analizar el control que ejercen las variables climáticas (temperaturas y precipitación) y los patrones espaciales de la aridez; y, v) para determinar el efecto antropogénico de los usos de suelos.

Todos estos estudios previos se han centrado en áreas donde existe una buena cobertura vegetal, y muy pocos informes han analizado las variaciones temproales y espaciales de la dinámica vegetal en áreas degradadas (cárcavas y zonas de riesgo de erosión), donde la vegetación es escaza. El término cárcavas es usado para describir áreas de depósitos o rocas pobremente consolidadas e intensamente afectadas por procesos de erosión superficial, donde la vegetación es escasa o ausente (GALLART et al., 2002). ALATORRE y BEGUERÍA (2009b) utilizaron técnicas de TLD para discriminar áreas de cárcavas y zonas de riesgo de erosión desarrolladas sobre margas Eocenas, en la cuenca del río Ésera (Pirineo español). La presencia de suelos desnudos y su gran tamaño permitió una buena discriminación mediante TLD. Sin embargo, las zonas de riesgo de erosión en torno a las cárcavas, coincidieron con las áreas de transición entre el suelo desnudo (cárcavas) y las zonas de matorrales o bosques, que se caracterizan por una escaza cobertura vegetal (10-50\%). Las cárcavas en zonas subhúmedas de montaña están sujetas a mayores tasas de denudación y una dinámica más activa en comparación con las zonas áridas o semiáridas (REGÜÉS \& GALLART, 2004). Por esta razón, el análisis de la dinámica vegetal sobre áreas con erosión activa (cárcavas) y zonas de riesgo de erosión es muy relevante para el diseño de medidas de 
mitigación y remediación de los efectos de la erosión y el transporte de sedimentos.

Los objetivos de este trabajo son: i) obtener una serie temporal de la actividad vegetal durante dos periodos (inicio de primavera y final de verano) para las distintas coberturas de suelo, incluyendo las zonas vegetadas y degradadas; ii) determinar el grado en que el clima está controlando la actividad vegetal en las distintas coberturas, y definir las tendencias temporales; y, iii) analizar la distribución espacial de las tendencias temporales de la actividad vegetal de las zonas de riesgo de erosión, como indicadores de recuperación o degradación, y cuantificar los efectos de diversos factores topográficos sobre dichas tendencias.

\section{2. Área de estudio}

El área de estudio se localiza aproximadamente a $23 \mathrm{~km}$ al $\mathrm{N}$ del embalse de Barasona, y está integrada por un corredor con afloramientos margosos eocenos de orientación NO-SE (Fig. 1A). El rango altitudinal va desde $620 \mathrm{~m}$ a $2149 \mathrm{~m}$ (Fig. 1B). La cartografía de las coberturas de suelo basada en el método de máxima verisimilitud (ALATORRE \& BEGUERÍA, 2009b) muestra que el área de estudio está ocupada principalmente por cinco categorías: cárcavas, $19 \mathrm{~km}^{2}$ (8.0\%); bosque de coníferas, $65 \mathrm{~km}^{2}$ (28.0\%); bosque de frondosas, $21 \mathrm{~km}^{2}(9.0 \%)$; pastizales, $32 \mathrm{~km}^{2}(13.0 \%)$; y matorrales, $99 \mathrm{~km}^{2}$ (42.0\%). La distribución espacial de las zonas ocupadas por matorrales y pastizales muestra que podrían ser clasificadas como cárcavas. Esta distribución espacial sugirió que existe una transición progresiva entre las zonas erosionadas y forestales (Fig. 1C). En el mismo estudio, se obtuvo un mapa de áreas con erosión activa (cárcavas) y zonas de riesgo de erosión, con $17 \mathrm{~km}^{2}$ y $49 \mathrm{~km}^{2}$, respectivamente (Fig. 1D). La comparación visual de los mapas muestra que las zonas de riesgo de erosión corresponden principalmente a la clase de matorral (y en algunos casos, pastizales y coníferas) que bordean las zonas de cárcavas (para más información por favor vea, ALATORRE \& BEGUERÍA, 2009b).

El clima es de montaña, húmedo y frío, con influencias atlánticas y mediterráneas continentales (GARCÍA-RUIZ et al., 1985). La temperatura media anual es de $11^{\circ} \mathrm{C}$, mientras que la precipitación se sitúa en torno a los $876 \mathrm{~mm}$ año ${ }^{-1}$. La precipitación se distribuye de manera irregular a lo largo del año, con un máximo en primavera de entre 180 y $160 \mathrm{~mm}$ (Abril y Mayo, respectivamente), y un período de precipitaciones mínimas en los meses de Julio y Agosto, en los que la precipitación no supera los $100 \mathrm{~mm}$. La topografía y la altitud explican las altas precipitaciones y las bajas 
temperaturas. Por encima de los $1700 \mathrm{~m}$ la precipitación llega a alcanzar los $2000 \mathrm{~mm}$ año ${ }^{-1}$; periodos sin lluvia son poco frecuentes y muy cortos,

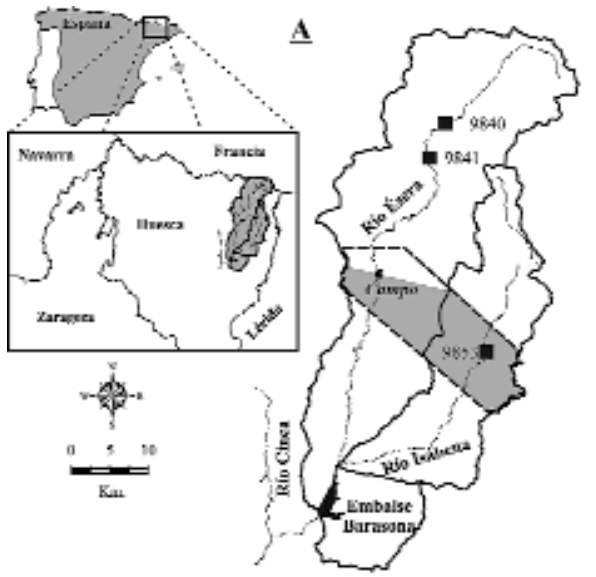

$\underline{B}$
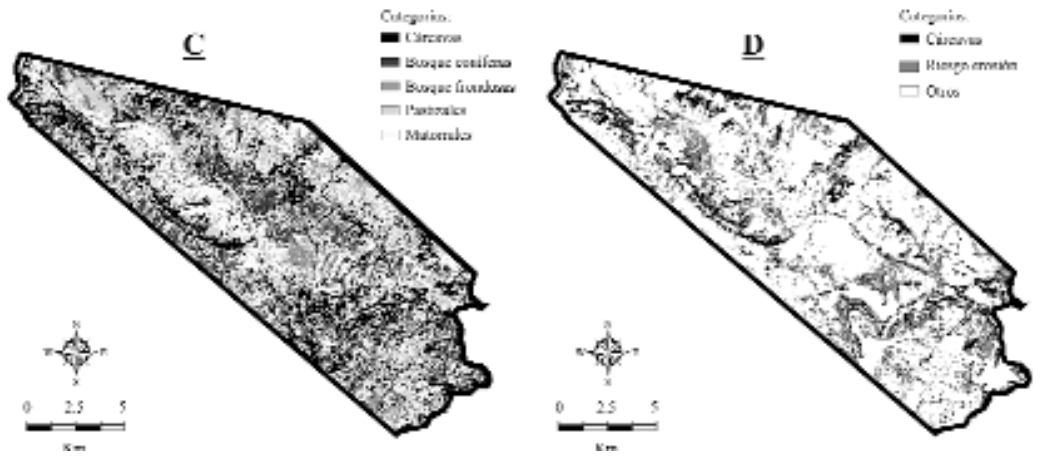

Figure 1. A) Localización del área de estudio: i) el área recortada indica la localización de áreas de cárcavas sobre margas Eocenas $\left(236 \mathrm{~km}^{2}\right)$; ii) la zona gris indica el área de la escena Landsat; iii) los cuadros negros indican la localización de las estaciones climatológicas de la Agencia Nacional de Meterología: Eriste (9840), Sesué (9841), Serraduy (9853). B) Modelo Digital del Terreno (MDT). C) Mapa de las coberturas de suelo basado en la clasificación supervisada usando el método de máxima verosimilitud y máxima regla de probabilidad de clasificación (Alatorre y Beguería, 2009b). D) Mapa de áreas con erosión activa (cárcavas) y zonas de riego de erosión (Alatorre y Beguería, 2009b).

Figure 1. A) Location of the study area: i) subset area indicates the location of badland areas on marls (236 $\mathrm{km} 2)$; ii) the gray zone indicates the area of the Landsat scene; iii) the black squares indicate the location of meteorological observatories of the National Agency of Meteorology: Eriste Central (9840), Sesué Central (9841), and Serraduy (9853). B) Digital terrain model (DTM). C) Land cover map based on supervised classification using the maximum likelihood method and the maximum probability classification rule (Alatorre and Begueria, 2009b). D) Erosion risk maps (Alatorre and Beguería, 2009b). 
resultando en breves periodos de déficit hídrico. Una gran parte de la precipitación cae como nieve, que persiste durante varios meses, ya que la isoterma de $0^{\circ} \mathrm{C}$ se encuentra a $1.650 \mathrm{~m}$.

\section{Datos y metodología}

\subsection{Selección de la base de datos y preparación}

En este trabajo se han utilizado una serie homogenizada de imágenes Landsat TM y ETM+ para el periodo 1984-2007. La base de datos comprende 28 imágenes, 12 de las cuales corresponden al inicio de primavera (Marzo) y 16 para finales del verano (Agosto). Las dos series temporales fueron usadas para identificar las posibles diferencias en la dinámica vegetal como una función estacional de la actividad vegetal, y para evaluar con mayor robustez los patrones temporales y espaciales de la actividad vegetal. La tabla 1 muestra la fecha de cada una de las imágenes empleadas en cada serie temporal. Detalles del procedimiento de corrección topográfica y atmosférica aplicado a las imágenes, y una descripción completa del conjunto de datos y su validación, han sido descritos por VICENTE-SERRANO et al. (2008).

Tabla 1. Datos de las imágenes Landsat 5 TM y 7 ETM+ usadas en el área de estudio. Table 1. Dates for the Landsat 5 TM and 7 ETM+ images used in the study.

\begin{tabular}{|cccc|}
\hline \multirow{2}{*}{ Fecha adquisición } & Sensor & Fecha adquisición & Agosto \\
\hline $03 / 11 / 1989$ & TM & $08 / 20 / 1984$ & TM \\
$03 / 30 / 1990$ & TM & $08 / 07 / 1985$ & TM \\
$03 / 06 / 1993$ & TM & $08 / 13 / 1987$ & TM \\
$03 / 09 / 1994$ & TM & $08 / 02 / 1989$ & TM \\
$03 / 28 / 1995$ & TM & $08 / 24 / 1991$ & TM \\
$03 / 17 / 1997$ & TM & $08 / 10 / 1992$ & TM \\
$03 / 20 / 1998$ & TM & $08 / 29 / 1993$ & TM \\
$03 / 23 / 1999$ & TM & $08 / 03 / 1995$ & TM \\
$03 / 17 / 2000$ & ETM + & $08 / 24 / 1997$ & TM \\
$03 / 10 / 2003$ & ETM+ & $08 / 14 / 1999$ & TM \\
$03 / 07 / 2005$ & TM & $08 / 08 / 2000$ & ETM + \\
$03 / 13 / 2007$ & TM & $08 / 26 / 2001$ & ETM+ \\
& & $08 / 30 / 2002$ & ETM + \\
& & $08 / 27 / 2004$ & TM \\
& & $08 / 18 / 2005$ & TM \\
& & $08 / 01 / 2006$ & TM \\
\hline
\end{tabular}


Las series temporales del NDVI fueron obtenidas a partir de las imágenes Landsat TM y ETM+, con el propósito de monitorizar la actividad vegetal. El NDVI fue calculado como (ROUSE et al., 1974):

$N D V I=\frac{\rho_{I R}-\rho_{R}}{\rho_{I R}+\rho_{R}}$

donde $\rho_{I R}$ es la reflectividad en la región del infrarrojo cercano del espectro electromagnético y $\rho_{R}$ es la reflectividad en la región del rojo. El NDVI es una medida de la capacidad fotosintética de las plantas (RUIMY et al., 1994) y la resistencia estomática con respecto a la transferencia de vapor de agua (TUCKER \& SELLERS, 1986). Por lo tanto, altos valores de NDVI son indicativos de la actividad de la vegetación secundaria.

También se ha utilizado el mapa de coberturas de suelo que comprende los principales tipos de vegetación en el área de estudio, así como el mapa de zonas con erosión activa (cárcavas) y áreas de riesgo de erosión (Fig. 1C y D; ALATORRE \& BEGUERÍA, 2009b).

Para analizar los efectos del clima sobre la actividad vegetal se utilizó una base de datos que consta de tres series de precipitación diaria de la Agencia Nacional de Meteorología, que incluye datos desde Enero de 1984 (Fig. 1A). Los datos diarios de temperatura se han obtenido para el mismo periodo de la estación Serraduy (Fig. 1A). Las series temporales de las precipitaciones totales y las temperaturas máximas / mínimas medias se calcularon a partir de la serie diaria original, sumando los valores diarios durante el periodo inmediatamente anterior a las fecha de cada imagen. Así, las series climáticas se calcularon para los siguiente periodos previos a la fecha de la imagen: 15 días, 30 días, 3 meses (Enero, Febrero y Marzo para las imágenes de Marzo, Junio, Julio y Agosto para las imágenes de Agosto) y 6 meses (Octubre a Marzo, y de Marzo a Agosto, respectivamente).

Las variables topográficas también fueron analizadas para evaluar sus efectos en la actividad vegetal. Para ello se utilizó un modelo digital del terreno (MDT) con una resolución espacial de $20 \mathrm{~m}$ para obtener la pendiente $\left(\mathrm{m} \mathrm{m}^{-1}\right)$, algunos estudios han demostrado la importancia de este factor para explicar las tasas de recuperación de la vegetación (Pueyo y Beguería, 2007). También, se derivó un modelo de radiación solar potencial $\left(\mathrm{MJ} \mathrm{m}^{-2}\right.$ día-1) para evaluar el control topográfico del balance de energía, utilizando un algoritmo que incluye el efecto de la complejidad del terreno (umbría y la reflexión) y la posición solar diaria (PONS \& NINYEROLA, 2008). 


\subsection{Análisis estadístico}

Las series temporales de NDVI para cada una de las coberturas de suelo fueron verificadas para determinar la presencia de tendencias temporales, usando la prueba de correlación de Spearman contra el tiempo. Esto permitió el análisis de la dinámica vegetal en términos de incremento (correlación positiva) y un descenso de la actividad (correlación negativa). El grado de significancia de las tendencias fue verificada usando el valor de $p$ asociado con el estadístico rho de Spearman.
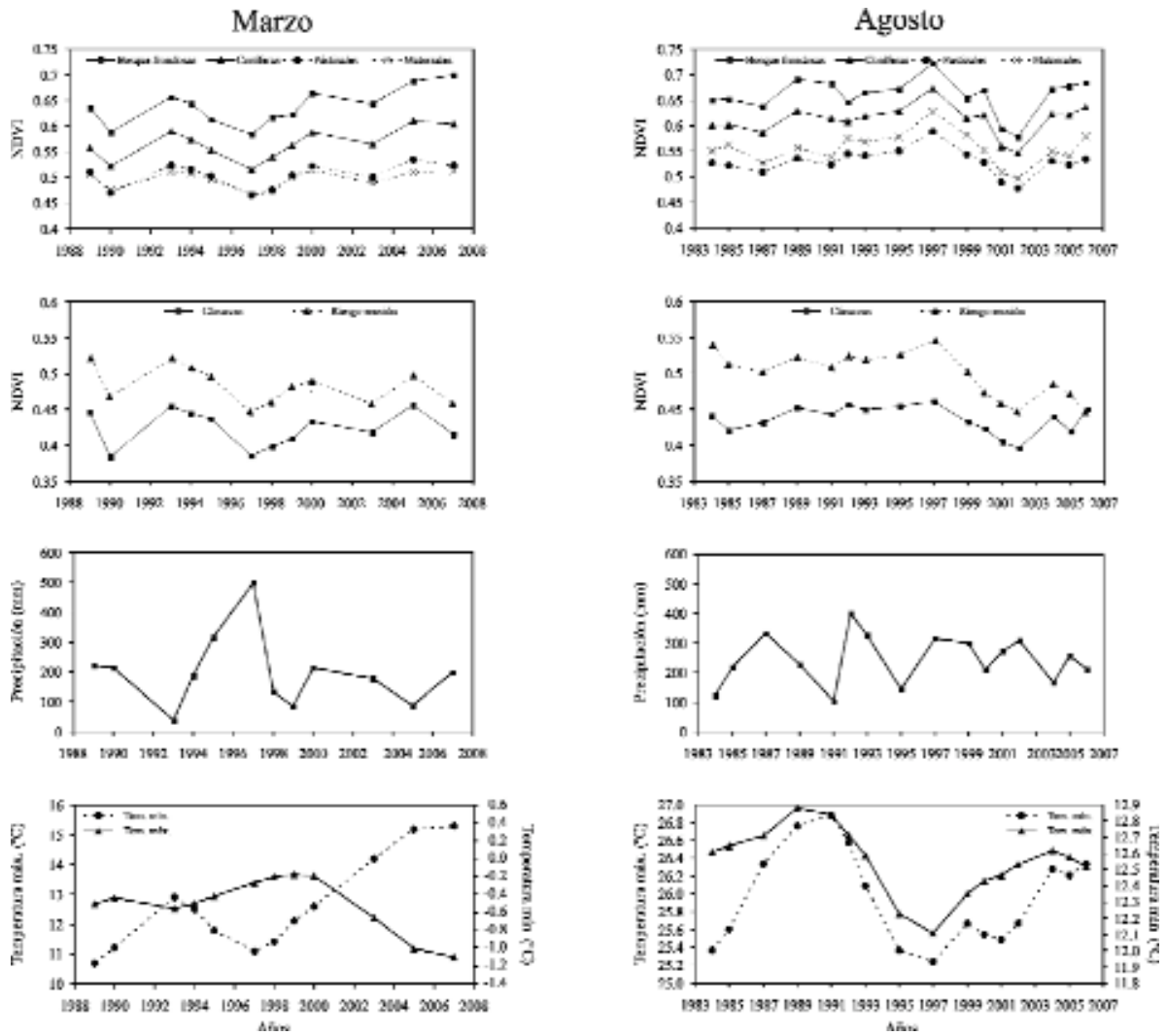

Figura 2. Evolución temporal de los valores medios del NDVI en Marzo y Agosto entre las clases de coberturas de suelo, cárcavas y zonas de riesgo de erosión.

Figure 2. Temporal evolution of the mean NDVI values for March and August between the categories of land cover map and erosion risk areas. 
La prueba de Spearman permite detectar la presencia de tendencias temporales en las series de NDVI, pero no identifica los factores implicados. Para determinar el control que ejerce el clima sobre la actividad vegetal y para aislarlo de otros factores, se realizó una regresión multivariante de los valores medios de NDVI contra las variables climáticas en cada una de las coberturas. Como paso intermedio se llevó acabo un análisis de la correlación para seleccionar la serie temporal climática más adecuada. Para las imágenes de Marzo y de Agosto se encontró que las series temporales climáticas que tenían una mayor correlación con el NDVI eran los 3 meses anteriores a la fecha de adquisición de cada imagen, las cuales fueron utilizadas como covariables en el análisis de regresión. También se introdujeron otras dos covariables, el día juliano y el año de adquisición de la imagen, ya que esto podría afectar el NDVI.

Para determinar las variables que explican significativamente la evolución temporal del NDVI sobre cada cobertura, se utilizó un procedimiento de retroceso "stepwise" basado en AIC (Akaike's information criterion statistic), implementado en software estadístico R (R Development Core Team, 2008).

Para realizar un análisis espacial, la regresión multivariante fue repetida píxel a píxel solo para las zonas de riesgo de erosión. Esto permitió obtener un mapa espacialmente distribuido de las tendencias del NDVI que no fueron explicadas por las variables climáticas, y así identificar las áreas que experimentan procesos de degradación o recuperación vegetal.

Finalmente, se realizó un análisis de correlación entre la evolución del NDVI y los factores topográficos (altitud, pendiente y la radiación solar potencial entrante), y un procedimiento "boostrap" para determinar la significación estadística de la correlación.

\section{Resultados y discusión}

\subsection{Evolución temporal del NDVI sobre las coberturas de suelo, 1984-2007}

La evolución temporal de los valores medios de NDVI en los meses de Marzo y Agosto para cada una de las cobertura de suelo fue evaluada (Fig. 2, Tabla 2). Se observó una clara diferencia entre las áreas vegetadas (bosque de coníferas y frondosas, pastizales y matorrales) y las áreas degradadas (cárcavas y áreas de riesgo de erosión). Las áreas vegetadas presentaron los valores de NDVI más altos, siendo los más elevados en Agosto. Los valores de NDVI en Marzo mostraron un tendencia temporal positiva para todas las áreas vegetadas, particularmente para los bosques de coníferas y frondosas donde las tendencias fueron más significativas $\alpha=0.05$. Sin embargo, el 
incremento del NDVI no fue constante, y en algunos años (p. ej. 1997 y 2003) se registró un descenso en los valores del NDVI con respecto a la tendencia general (Fig. 2). Los valores de NDVI en el mes de Agosto no mostraron una tendencia temporal significativa en ninguna de las áreas vegetadas. Estos resultados sugieren un incremento en la actividad vegetal durante el periodo de estudio asociado a condiciones más favorables.

Las áreas degradadas (cárcavas y zonas de riesgo de erosión) mostraron los valores medios de NDVI más bajos, con una ligera diferencia entre Marzo y Agosto ocasionada por la escasa cobertura vegetal (Tabla 2). Las tendencias del NDVI fueron negativas para Marzo y Agosto, y fueron más acusadas en las áreas de riesgo de erosión, encontrándose que la serie temporal de Agosto es estadísticamente significativa. Esto podría indicar la presencia de procesos de degradación (erosión acelerada) en estas áreas.

Tabla 2. Valores medios del NDVI y tendencias temporales del NDVI (correlación de Spearman rho contra el tiempo y significación estadística) en cada una de las clases de coberturas en Marzo y Agosto.

Table 2. NDVI values and temporal NDVI trends (Spearman's rho correlation with time and significance) for each land use category for March and August.

\begin{tabular}{|lccccccccc|}
\hline & \multicolumn{4}{c}{ Marzo } & \multicolumn{4}{c|}{ Agosto } \\
Cobertura de suelo & \multicolumn{3}{c}{ NDVI } & \multicolumn{3}{c|}{ Tendencia NDVI } & \multicolumn{2}{c|}{ NDVI } & Tendencia NDVI \\
& media & desvest & rho & $p$ & media & desvest & rho & $p$ \\
\hline Bosque frondosas & 0,63 & 0,10 & 0,517 & 0,0862 & 0,65 & 0,11 & 0,321 & 0,224 \\
Bosque coníferas & 0,56 & 0,12 & 0,573 & 0,0538 & 0,61 & 0,12 & 0,168 & 0,520 \\
Pastizales & 0,49 & 0,11 & 0,336 & 0,281 & 0,55 & 0,15 & $-0,0265$ & 0,926 \\
Matorrales & 0,50 & 0,11 & 0,294 & 0,348 & 0,52 & 0,13 & $-0,0899$ & 0,741 \\
Zonas de riesgo de erosión & 0,48 & 0,11 & $-0,196$ & 0,543 & 0,50 & 0,14 & $-0,594$ & 0,0173 \\
Cárcavas & 0,42 & 0,12 & $-0,0420$ & 0,904 & 0,41 & 0,16 & $-0,250$ & 0,349 \\
\hline
\end{tabular}

Estos resultados sugieren que la presencia de tendencias temporales opuestas en el área de estudio depende de la naturaleza de cada cobertura, con áreas vegetadas que experimentan un incremento en la actividad vegetal y áreas degradadas que sufren una disminución de la cubierta vegetal. Sin embargo, la variabilidad temporal del NDVI puede explicarse por las condiciones climáticas, como se verá en la siguiente sección.

\subsection{Análisis de la regresión del NDVI contra las variables climáticas}

El análisis de la regresión permitió explicar los patrones temporales del NDVI observados sobre cada cobertura de suelo. El modelo de regresión en 
general se ajustó bien a los valores de NDVI observados, solo en el caso de los pastizales, cárcavas y las zonas de riesgo de erosión en el mes de Marzo, los resultados de modelo estuvieron ligeramente por debajo del nivel de confianza (Tabla 3). El mejor ajuste se obtuvo en Marzo para las zonas con buena cobertura vegetal (Bosques de coníferas y frondosas), no de la misma forma para las áreas degradadas (pastizales, cárcavas y áreas de riesgo de erosión), como lo demuestra los bajos de $\mathrm{R}^{2}$. En Agosto, la bondad de ajuste fue similar para todas las coberturas (Tabla 4). En todos los modelos fueron identificadas una o más variables climáticas que explican la evolución temporal de la actividad vegetal.

Tabla 3. Análisis de la regression de los valores de NDVI para Marzo en relación con las condiciones climáticas.

Table 3. Regression analysis of NDVI values for March in relation to climatic conditions.

\begin{tabular}{|lcccccc|}
\hline & $\begin{array}{c}\text { Bosque } \\
\text { coniferas }\end{array}$ & $\begin{array}{c}\text { Bosque } \\
\text { frondosas }\end{array}$ & Matorrales & Pastizales & $\begin{array}{c}\text { Riesgo } \\
\text { erosión }\end{array}$ & $\begin{array}{c}\text { Erosión } \\
\text { (cárcavas) }\end{array}$ \\
\hline $\mathrm{R}^{2}$ & 0,743 & 0,779 & 0,615 & 0,424 & 0,467 & 0,547 \\
$\mathrm{p}$ & 0,002 & 0,001 & 0,045 & 0,084 & 0,150 & 0,082 \\
Error estandar residual & 0,561 & 0,520 & 0,728 & 0,839 & 0,856 & 0,789 \\
Beta coeficiente: & & & & & & - \\
$\quad$ Precipitacióm & $-0,317$ & - & $-0,298$ & - & - & - \\
$\quad$ T max & - & - & - & - & - & - \\
$\quad$ T min & 0,683 & 0,678 & 0,371 & 1,11 & 0,701 & 0.716 \\
$\quad$ Día juliano & - & $-0,310$ & $-0,326$ & - & $-0,377$ & -0.457 \\
$\quad$ Tiempo (año) & - & - & - & $-0,705$ & $-0,845$ & -0.719 \\
Tendencia temporal & & & & & & \\
(cambio en NDVI): & - & - & - & $-0,00216$ & $-0,00433$ & -0.00326 \\
$\quad$ Por año & - & - & - & $-4,03 \%$ & $-7,91 \%$ & $-6.02 \%$ \\
$\quad$ Periodo 1989-2007 & - & & & & &
\end{tabular}

Sin embargo hubo diferencias entre Marzo y Agosto, así como entre las distintas clases de coberturas. En Marzo, la temperatura mínima media fue el factor más explicativo, como lo demuestra el hecho del mayor coeficiente beta (estandarizado). Esto refleja la importancia de un clima relativamente cálido a finales de invierno/comienzos de primavera, cuando empieza el periodo de crecimiento. La temperatura máxima media no explicó ninguna de las tendencias de NDVI observadas en las distintas clases de coberturas, la precipitación acumulada fue significativa en solo unos pocos casos (bosque de coníferas y frondosas). Contrariamente a lo esperado, la precipitación acumulada tuvo un efecto negativo en el índice de vegetación (es decir, una 
mayor precipitación se tradujo en valores bajos de NDVI), como lo demuestran los signos negativo de los coeficientes beta. El momento de la adquisición de la imagen (variable = "día") fue estadísticamente significativo en el mes de Marzo en todas las coberturas, excepto para las coberturas de bosques de coníferas y pastos, lo que sugiere la importancia del estado fenológico de la vegetación en esa época del año.

Tabla 4. Análisis de la regression de los valores de NDVI para Agosto en relación con las condiciones climáticas.

Table 4. Regression analysis of NDVI values for August in relation to climatic conditions.

\begin{tabular}{|lcccccc|}
\hline & $\begin{array}{c}\text { Bosque } \\
\text { coniferas }\end{array}$ & $\begin{array}{c}\text { Bosque } \\
\text { frondosas }\end{array}$ & Matorrales & Pastizales & $\begin{array}{c}\text { Riesgo } \\
\text { erosión }\end{array}$ & $\begin{array}{c}\text { Erosión } \\
\text { (cárcavas) }\end{array}$ \\
\hline $\mathrm{R}^{2}$ & 0,599 & 0,591 & 0.663 & 0.640 & 0.681 & 0.663 \\
$\mathrm{p}$ & 0,028 & 0,031 & 0.004 & 0.005 & 0.003 & 0.004 \\
Error estandar residual & 0,739 & 0,747 & 0.649 & 0.671 & 0.632 & 0.649 \\
Beta coeficiente: & & & & & & \\
$\quad \begin{array}{l}\text { Precipitacióm } \\
\quad \text { T max }\end{array}$ & $-0,325$ & $-0,421$ & - & - & - & - \\
$\quad$ T min & $-1,59$ & $-1,45$ & -1.66 & -1.62 & -1.23 & -1.50 \\
$\quad$ Día juliano & 1,31 & 1.34 & 1.21 & 1.09 & 0.806 & 1.42 \\
$\quad$ Tiempo (año) & $-0,481$ & -0.507 & -0.688 & -0.646 & -1.000 & -0.789 \\
Tendencia temporal & & & & & & \\
(cambio en NDVI): & & & & & & \\
$\quad$ Por año & $-0,00197$ & -0.00247 & -0.00241 & -0.00244 & -0.00363 & -0.00210 \\
$\quad$ Periodo 1989-2007 & $-4,43 \%$ & $-5.53 \%$ & $-5.40 \%$ & $-5.46 \%$ & $-8.02 \%$ & $-4.72 \%$ \\
\hline
\end{tabular}

En Agosto la temperatura mínima media también fue estadísticamente significativa (positiva) para todas las clases de coberturas, pero el factor más importante fue la temperatura máxima media. Esto lo demostró el coeficiente beta absoluto más alto, y un efecto negativo en todos los casos, lo que significa que un verano cálido se traduce en valores más bajos del NDVI. La precipitación acumulada fue significativa solo para los bosques de coníferas y frondosas, donde también tuvo un efecto negativo en el NDVI. Mientras que el momento de adquisición de la imagen no tuvo ningún efecto significativo para cualquiera de las coberturas.

Una vez que se han explicado los efectos climáticos y fenológicos del NDVI, se procedió a identificar las tendencias temporales de los valores medios del NDVI para algunas de las clases de coberturas de suelo (Tabla 3 y 4). En Marzo se encontraron tendencias negativas del NDVI solo para pastizales, cárcavas y áreas de riesgo de erosión, lo que representa una 
disminución del NDVI, aproximadamente entre 4-8\% en el periodo comprendido entre 1989-2007. En cambio, en Agosto todas las coberturas mostraron una tendencia temporal negativa, y mostraron un rango similar en el periodo de 1984-2006. Las magnitudes de las tendencias negativas fueron similares para todas las clases de coberturas, con una excepción. Las zonas de riesgo de erosión mostraron los valores más altos (aproximadamente 8\%) tanto en Marzo como en Agosto.

El análisis de los resultados de la regresión permitió la interpretación de las tendencias temporales del NDVI (Fig. 2). La tendencias del NDVI al alza se presentaron en las áreas con buena cobertura vegetal, en el caso de Marzo se ha explicado por una tendencia similar en las temperaturas mínimas medias. La tendencia a la baja del NDVI en las zonas de riesgo de erosión también fue evidente, pero no pudo ser correlacionada con la evolución temporal de las variables climáticas.

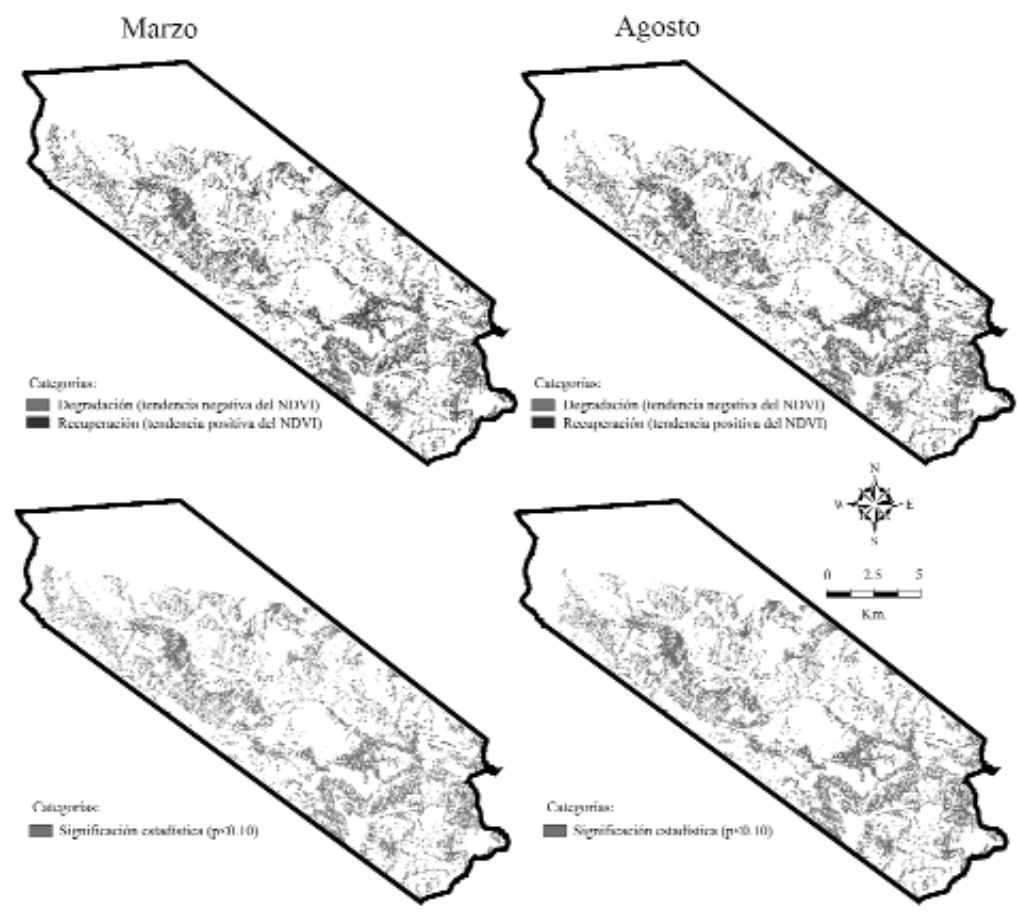

Figura 3. Distribución espacial de las tendencias del NDVI para Marzo y Agosto en las zonas de riesgo de erosión después de quitar la influencia de clima, encontrando: signo de la tendencia temporal (arriba) y significación estadística (abajo).

Figure 3. Spatial distribution of the NDVI trends for March and August in erosion risk areas after climatic forcing was accounted for: sign of temporal trend (above) and significance (below). 
Estos resultados están muy relacionados con la evolución observada en Pirineo occidental español. VICENTE-SERRANO et al. (2004) encontraron tendencias positivas del NDVI en bosques y en áreas con vegetación bien desarrollada, lo cual fue relacionado con un aumento en la temperatura media anual, y a los patrones de abandono de las tierras y los procesos naturales de colonización vegetal (LASANTA \& VICENTE-SERRANO, 2007). En el presente estudio también se encontró una tendencia positiva del NDVI en las áreas vegetadas, y se demostró que la temperatura máxima y mínima media registrada en los 3 meses previos a la toma de las imágenes Landsat ejerció una influencia opuesta a la del NDVI, y que este efecto varió durante el año.

Encontrar que la precipitación acumulada tuviera un efecto negativo sobre el NDVI fue desconcertante, ya que se esperaba un efecto positivo. Esta anomalía puede ser explicada por los siguientes factores: i) la disponibilidad del agua no constituye un factor limitante para el crecimiento de la vegetación en el área de estudio, la cual recibe un promedio de $900 \mathrm{~mm}$ año${ }^{1}$, principalmente en invierno y primavera; y ii) la cantidad de precipitación está bien correlacionada con la cobertura de nubes en la región, con periodos de lluvia que resultan en una reducción de la radiación solar entrante, que se eleva en los días claros. Es bien sabido que el nivel de precipitación deja de ser un factor limitante para el crecimiento de la vegetación en regiones húmedas, donde la competencia por el espacio y la radiación solar es más importante. Varios estudios han documentado la saturación del NDVI por efecto de la precipitación en zonas húmedas (SANTOS \& NEGRÍN, 1997). La relación no lineal entre las precipitaciones y la actividad de la vegetación en estas regiones podría ser explicada por la ausencia de un efecto positivo significativo, pero no la presencia de un efecto negativo, como en nuestra área de estudio. Sin embargo, la segunda explicación (nubosidad) podría facilitar la explicación de nuestros datos. Lamentablemente, se carece de la disponibilidad de series temporales de nubosidad, por lo que la hipótesis no pudo ser probada.

En estudios a escala más local, se han observado tendencias temporales similares del NDVI, pero el impacto humano ha sido incluido entre las explicaciones. FULLER (1998) analizó una serie temporal de 7 años de NDVI en Senegal para el periodo 1987-1993, y observó las diferencias espaciales en las tendencias temporales del NDVI en función a las prácticas agrícolas y la gestión de pastos. PELKEY et al. (2000) indicaron que, en Tanzania, la creación de áreas naturales protegidas estaba teniendo un efecto positivo en el incremento de la cobertura vegetal y la biomasa en relación con las áreas que muestra un uso humano más intensivo. La presencia de una tendencia residual en los valores medios del NDVI tras la eliminación de las influencias climáticas se suele considerar que constituye una evidencia de que otros 
factores, tales como las prácticas de uso humano del suelo, están afectando a la actividad vegetal. En el presente estudio se ha encontrado en Agosto tendencias estadísticamente significativas para todas las cuberturas. Dada la baja intensidad del uso del suelo en la región, la atribución de estas tendencias a causas humanas es difícil. Además, las tendencias positivas del NDVI en Marzo fueron explicadas por evolución de las temperaturas mínimas. Por lo tanto, el adelantamiento del periodo de crecimiento, además del aumento en la actividad vegetal durante primavera y principios de verano, pode causar mayor estrés en la vegetación en el mes de Agosto. Tras la eliminación de los efectos climáticos, las tendencias del NDVI en Marzo y Agosto fueron a la baja tanto en pastizales como en las áreas de cárcavas, en particular en las zonas de riesgo de erosión. Esto puede constituir un signo de degradación de estas zonas debido a la disminución de la actividad vegetal en el área de estudio como efecto del proceso de erosión del suelo en estas zonas (ALATORRE \& BEGUERÍA, 2009b). En las zonas de erosión el efecto de la tendencia temporal fue mayor que el efecto de las variables climáticas y, en consecuencia, estas áreas muestran en general una tendencia a la baja del NDVI. Como esta clase de cobertura incluye zonas muy sensibles que corren riesgo de perder toda la cobertura vegetal, convirtiéndose así en cárcavas, nos hemos centrado más en los factores que han contribuido a esta degradación.

\subsection{Distribución espacial de las tendencias positivas y negativas del NDVI en zonas de riesgo de erosión}

Las tendencias negativas del NDVI en las zonas de riesgo de erosión observadas en Marzo y Agosto, no pudieron ser explicadas por los factores climáticos, lo cual sugirió que los procesos de degradación como la erosión o la expansión lateral de las cárcavas eran los responsables. Esta posibilidad motivó una evaluación detallada de la distribución espacial de las tendencias del NDVI en las zonas de riesgo de erosión.

Tras eliminar las influencias climáticas, la distribución espacial de las tendencias positivas y negativas del NDVI en las zonas de riesgo de erosión fue similar en Marzo y Agosto, lo que demuestra que el proceso es bastante consistente, y no solo atribuible a los efectos estacionales (Fig. 3). Las tendencias negativas del NDVI predominaron en las dos imágenes, lo que indica las ocurrencias de procesos de degradación en estas áreas. Sin embargo, existen regiones donde predominan las tendencias positivas, sobre todo en la imagen de Marzo. La proporción de las tendencias estadísticamente significativas es mayor en Agosto, debido al aumento de las condiciones de estrés, que predominan en este mes. 
El mapa de la distribución espacial de las tendencias del NDVI píxel a píxel permitió evaluar el efecto de determinadas condiciones topográficas sobre la presencia de procesos de degradación o recuperación vegetal. En Marzo, se encontró una relación positiva entre la evolución del NDVI y la elevación pero estadísticamente no significativa $(p=0.283)$ (Fig. 4). Esto puede ser relacionado con la localización de las áreas de cárcavas; estas predominan en el fondo de la depresión Eocena, en contraste con las zonas boscosas, que se encuentran principalmente en las laderas. Se encontró una correlación negativa entre la evolución del NDVI y la pendiente, lo que sugiere una asociación de pendientes más pronunciadas con las tendencias negativas. Esta asociación podría ser relacionada con la influencia positiva que tiene la pendiente con la actividad de procesos de erosión. Se obtuvieron resultados similares con las imágenes de Agosto (Fig. 4), aunque las relaciones con la altitud y la pendiente fueron más débiles $(p=0.447$ y $p=$ 0.416).
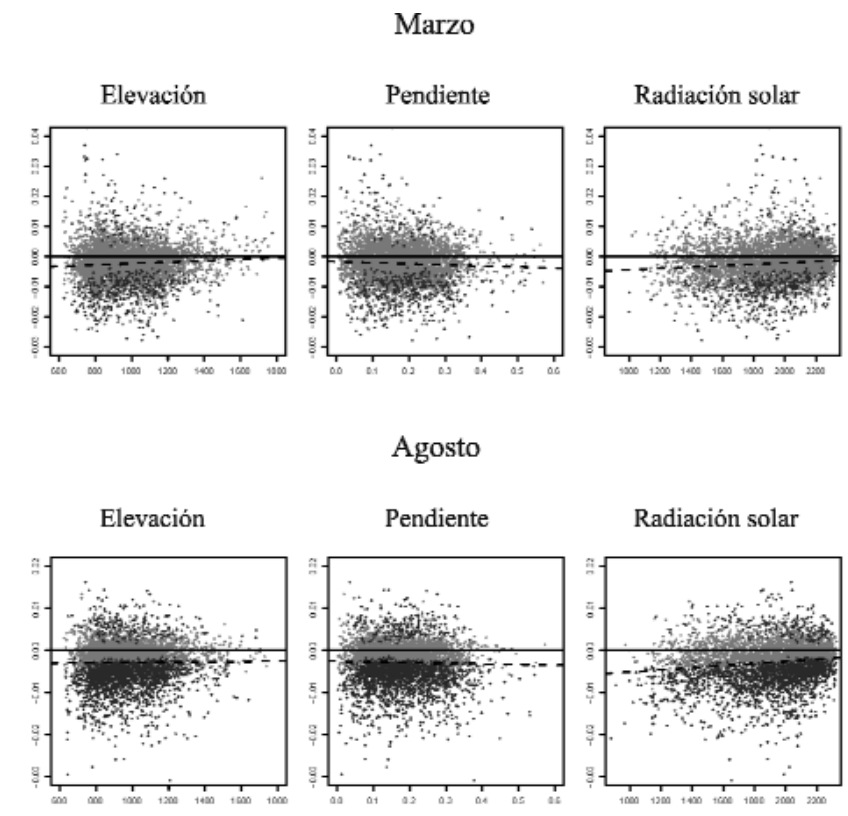

Figura 4. Correlación entre las tendencias de NDVI para Marzo y Agosto en las zonas de riesgo de erosión y las variables topográficas (después de quitar la influencia del clima). Los resultados contienen una muestran aleatoria del 10\% de los píxeles originales. Los puntos negros indican los píxeles con una tendencia estadísticamente significativa.

Figure 4. Correlation between the NDVI trends for March and August in the erosion risk areas and topographic variables (after climatic forcing was accounted for). Results are shown for a random sample containing $10 \%$ of the original pixels. The black dots indicate pixels with statistically significant trends. 
Con respecto a la radiación solar potencial, tanto en Marzo como en Agosto se encontraron correlaciones positivas con las tendencias del NDVI ( $p$ $=0.113$ y $p=0.0345$, respectivamente), lo que sugiere que los procesos de degradación se producen preferentemente en las zonas de umbría (orientación norte). Esto resulta consistente en otras investigaciones que han revelado que las áreas de cárcavas se presentan predominantemente en las laderas de umbría (ALATORRE \& BEGUERÍA, 2009b) y están asociadas a los procesos mecánicos de meteorización por efecto de los ciclos de hielo y deshielo, que son más frecuentes en las laderas con orientación norte (NADAL-ROMERO et al., 2007).

Estos resultados contrastan con estudios en el Pirineo español que se han centrado en el control que ejerce la topografía sobre la recuperación vegetal, que han demostrado que en las laderas de umbría se registran mayores tasas de recuperación que en las laderas soleadas (LASANTA et al., 2000; VICENTE-SERRANO et al., 2004; LASANTA \& VICENTE-SERRANO, 2006; PUEYO \& BEGUERÍA, 2007). Esto ha sido explicado por la presencia de un mayor estrés sobre la vegetación causado por el aumento de la evapotranspiración en las laderas soleadas. Los resultados del presente estudio muestran que la influencia topográfica sobre los procesos de recuperación vegetal es diferente en zonas de riesgo de erosión, asociada a los procesos de erosión, que en las zonas con buena cubierta vegetal

\section{Conclusiones}

Se ha analizado la evolución temporal de la actividad vegetal sobre áreas vegetadas y en zonas de riesgo de erosión en una pequeña área del Pirineo central durante el periodo 1984-2007. Se obtuvieron dos series temporales de NDVI a partir de una serie homogenizada de imágenes Landsat TM y ETM+ para los meses de Marzo y Agosto. Esto permitió el análisis de la dinámica espacial y temporal de la actividad vegetal en áreas con buena cobertura vegetal (bosques y matorrales densos) y en zonas degradadas afectadas por procesos de erosión (cárcavas y zonas de riesgo de erosión). Las tendencias temporales del NDVI fueron analizadas mediante una regresión multivariante incorporando la evolución temporal de los factores climáticos (precipitación y la temperatura máxima y mínima).

Se esperaban diferencias estacionales en los patrones espaciales de la actividad vegetal o de los procesos de recuperación vegetal, como una consecuencia de la estacionalidad climática en la región y de la gran diferencia de disponibilidad de agua en primavera y verano (la vegetación en esta última estación es comúnmente afectada por estrés hídrico). 
Los resultados obtenidos podrían haber sido afectados por la heterogeneidad y la naturaleza de las coberturas seleccionadas, ya que esta zona montañosa es compleja y presenta una gran diversidad espacial. Sin embargo, la resolución espacial $(30 \mathrm{~m})$ de la imagen Landsat permitió una buena representación de las distintas coberturas seleccionadas. La asignación a cada cobertura de la clase más representativa, por superficie, en un tamaño de píxel de $30 \mathrm{~m}$ podría introducir algunos errores, pero era necesario garantizar una comparación eficaz entre el conjunto de datos de NDVI y la información categórica. Por otra parte, los resultados fueron espacialmente coherentes, claros y las pautas del NDVI coincidieron con la distribución espacial de las distintas coberturas de suelo empleadas.

En resumen, este estudio demostró que, en una zona montañosa representativa del Pirineo central español, se ha producido en los últimos 24 años un significativo incremento en la actividad vegetal, que se explica por el aumento de la temperatura mínima. Los bosques de coníferas y frondosa han registrado el mayor aumento en la actividad vegetal, mientras que en los pastizales y matorrales ha sido moderado. Sin embargo, las condiciones extremas que existen en las áreas con erosión activa y zonas de riesgo de erosión no han permitido la recuperación de la vegetación en el periodo de estudio.

\section{Agradecimientos}

La investigación realizada se enmarca dentro del proyecto PROBASE: "Procesos y balances hidrológicos y de sedimentos a diferentes escalas espaciales en ambientes mediterráneos: efectos de las fluctuaciones climáticas y los cambios de uso del suelo" (CONSOLIDER, CGL2006-11619/HID). La contribución del primer autor ha sido posible gracias a la beca predoctoral otorgada por el Consejo Nacional de Ciencia y Tecnología de México (CONACYT).

\section{Referencias}

ALATORRE, L.C. \& BEGUERÍA, S., 2009a. Los modelos de erosión: una revisión. Cuaternario \& Geomorfología, 23(1-2): 43-62.

ALATORRE, L.C. \& BEGUERÍA, S., 2009b. Identification of eroded areas using remote sensing in a badlands landscape on marls in the central Spanish Pyrenees. Catena, 76: 182-190. 
BEGUERÍA, S., LÓPEZ-MORENO, J.I., LORENTE, A., SEEGER, M. \& GARCÍA-RUIZ, J.M., 2003. Assessing the effect of climate oscillations and land-use change on streamflow in the central Spanish Pyrenees. Ambio, 32(4): 283-286.

DEFRIES, R., HOUGHTON, R.A., HANSEN, M.C., FIELD, C.B., SKOLE, D. \& TOWNSHEND, J., 2002. Carbon emissions from tropical deforestation and regrowth based on satellite observations for the 1980s and 1990s. Proceding of the Nacional Academic of Sciences of the United States of America, 99: 1425614261.

DELBART, N., LE TOAN, T., KERGOAT, L. \& FEDOTOVA, V., 2006. Remote sensing of spring phenology in boreal regions: A free of snow-effect method using NOAA-AVHRR and SPOT-VGT data (1982-2004). Remote Sensing of Environment, 101: 52-62.

DELBART, N., PICARD, G., LE TOAN, T., KERGOAT, L., QUEGAN, S., WOODWARD, I., DYE, D. \& FEDOTOVA, V., 2008. Spring phenology in boreal Eurasia in a nearly century time-scale. Global Change Biology, 14 (3): 603-614.

FULLER, D.O., 1998. Trends in NDVI time series and their relation to rangeland and crop production in Senegal, 1987-1993. International Journal of Remote Sensing, 19: 2013-2018.

GALLART, F., LLORENS, P., LATRON, J. \& REGÜÉS, D., 2002. Hydrological processes and their seasonal controls in a small Mediterranean mountain catchment in the Pyrenees. Hydrology and Earth System Sciences, 6(3). 527537.

GARCÍA-RUIZ, J.M., PUIGDEFÁBREGAS, J. \& CREUS, J., 1985. Recursos hídricos Superficiales del Alto Aragón. Instituto de Estudios Altoaragoneses, 224 pp., Huesca.

KIRKBY, M.J., IMESON, A.C., BERGKAMP, G. \& CAMMERAAT, L.H., 1996. Scaling up processes and models from the field plot to the watershed and regional areas. Journal of Soil and Water Conservation, 51: 391-396.

LAMBIN, E.F., 1996. Change detection at multiple temporal scales: seasonal and annual variations in landscape variables. Photogrammetric Engineering and Remote Sensing, 62(8): 931-938.

LASANTA, T., VICENTE-SERRANO, S. \& CUADRAT, J.M., 2000. Marginación productiva y recuperación de la cubierta vegetal en el Pirineo: un caso de estudio en el Valle de Borau. Boletín de la Asociación de Geógrafos Españoles, 29: 5-28.

LASANTA, T., \& VICENTE-SERRANO, S., 2006. Factores en la variabilidad espacial de los cambios en la cubierta vegetal en el Pirieno. Cuadernos de Investigación Geográfica, 32: 57-80. 
LASANTA, T. \& VICENTE-SERRANO, S., 2007. Cambios en la cubierta vegetal en el Pirieno Aragonés en los últimos 50 años. Pirineos, 162: 125-154.

LUCHT, W., PRENTICE, I.C., MYNENI, R.B., SITCH, S., FRIEDLINGSTEIN, P., CRAMER, W., BOUSQUET, P., BUERMANN, W. \& SMITH, B., 2002. Climatic control of the high-latitude vegetation greening trend and Pinatubo effect. Science, 296: 1687-1689.

MARTÍNEZ-VILLALTA, J., LÓPEZ, B.C., ADELL, N., BADIELLA, L. \& NINYEROLA M., 2008. Twentieth century increase of Scots pine radial growth in NE Spain shows strong climate interactions. Global Change Biology, 14(12): 2868-2881.

NADAL-ROMERO, E., REGÜÉS, D., MARTÍ-BONO, C. \& SERRANOMUELA, P., 2007. Badlands dynamics in the Central Pyrenees: temporal and spatial patterns of weathering processes. Earth Surfaces Processes and Landforms, 32(6): 888-904.

OLSSON, E., AUSTRHEIM, G. \& GRENNE, S.N., 2000. Landscape change patterns in mountains, land use and environmental diversity, MidNorway 1960-1993. Landscape Ecology, 15: 155-157.

PELKEY, N.W., STONER, C.J. \& CARO, T.M., 2000. Vegetation in Tanzania: assessing long term trends and effects of protection using satellite imaginery. Biological Conservation, 94: 297-309.

PONS, X. \& NINYEROLA, M., 2008. Mapping a topographic global solar radiation model implemented in a GIS and refined with ground data. International Journal of Climatology, 28: 1821-1834.

PUEYO, Y. \& BEGUERÍA, S., 2007. Modelling the rate of secondary succession after farmland abandonment in a Mediterranean mountain area. Landscape and Urban Planning, 83(4): 245-254.

R Development Core Team, 2008. R: A Language and Environment for Statistical Computing, Vienna (Austria), R Foundation for Statistical Computing.

REGÜÉS, D. \& GALLART, F., 2004. Seasonal patterns of runoff and erosion responses to simulated rainfall in a badland area in Mediterranean mountain conditions (Vallcebre, southeastern Pyrenees). Earth Surface Processes and Landforms, 29: 755-767.

RIAÑO, D., RUIZ, J.A.M., ISIDORO, D. \& USTIN, S.L., 2007. Global spatial patterns and temporal trends of burned area between 1981 and 2000 using NOAA-NASA Pathfinder. Global Change Biology, 13: 40-50.

ROUSE, J.W., HASS, R.H., SCHELL, J.A., DEERING, D.W. \& HARLAN, J.C., 1974. Monitoring the vernal advancement and retrogradation (greenwave effect) of natural vegetation. NASA/GSFC type III final report, Greenbelt, M.D.

RUIMY, A., SANGIER, B. \& DEDIU, G., 1994. Methodology for the estimation of terrestrial primary production from remotely sensed data. Journal of Geophysical Research, 99(D3): 5263-5283. 
SANTOS, P. \& NEGRÍN, A.J., 1997. A comparison of the Normalized Difference Vegetation Index and rainfall for the Amazon and Notheastern Brazil. Journal of Climate, 36: 958-965.

TUCKER, C.J. \& SELLERS, P., 1986. Satellite remote Sensing of primary production. International Journal of Remote Sensing, 7: 1395-1416.

VICENTE-SERRANO, S.M., LASANTA, T. \& ROMO, A., 2004. Analysis of the spatial and temporal evolution of vegetation cover in the Spanish central Pyrenees: the role of human management. Environmental Management, 34: 802-818.

VICENTE-SERRANO, S., BEGUERÍA, S. \& LASANTA, T., 2006a. Diversidad espacial de la actividad vegetal en campos abandonados del Pirineo español: Análisis de los procesos de sucesión mediante imágenes Landsat (1984-2001). Pirineos, 161: 59-84.

VICENTE-SERRANO, S.M., CUADRAT-PRATS, J.M. \& ROMO, A., $2006 \mathrm{~b}$. Aridity influence on vegetation patterns in the middle Ebro Valley (Spain): Evaluation by means of AVHRR images and climate interpolation techniques. Journal of Arid Environments, 66: 353-375.

VICENTE-SERRANO, S.M., PÉREZ-CABELLO, F. \& LASANTA, T., 2008. Assessment of radiometric correction techniques in analyzing vegetation variability and change using time series of Landsat images. Remote Sensing of Environment, 112: 3916-3934.

VRIELING, A., STERK, G. \& VIGIAK, O., 2006. Spatial evaluation of soil erosion risk in theWest Usambara Mountains, Tanzania. Land Degradation and Development, 17(3): 301-319.

WARREN, A., 2002. Land degradation is contextual. Land Degradation and Development, 13: 449-459.

ZENG, J., NEELIN, D., LAN, K.M. \& TUCKER, C.J., 1999. Enhancement of interdecadal climate variability in the Sahel by vegetation interaction. Science, 286: 1537-1540. 